\title{
Surveillance colonoscopy in patients with sessile serrated adenoma
}

\author{
Ji Hyung Nam, Hyoun Woo Kang \\ Department of Internal Medicine, Dongguk University Ilsan Hospital, Dongguk University College of Medicine, Goyang, Korea
}

I read the scientifically significant paper by Park et al. ${ }^{1}$ titled "Clinical outcomes of surveillance colonoscopy for patients with sessile serrated adenoma," which described endoscopic findings of sessile serrated adenoma (SSA) and clinicopathological results on surveillance colonoscopy for those patients. The authors showed that approximately two-thirds of SSAs were found in the proximal colon during complete colonoscopy, and that $5.8 \%$ of SSA patients were confirmed to have synchronous colorectal cancer (CRC) at baseline colonoscopy. Meanwhile, no metachronous cancer was detected during follow-up surveillance. The study highlighted the need for meticulous examination of the proximal colon to detect SSAs and confirmed that at least annual colonoscopy surveillance is not necessary in such patients.

In the past decade, the sessile serrated lesion has been an engaging topic of scientific interest as a premalignant lesion of CRC, and is known to be associated with interval cancer.

There is confusion about the nomenclature and pathologic criteria for a serrated lesion. The most commonly used terminology is that of the World Health Organization (WHO), ${ }^{2}$ which has adopted the term "sessile serrated adenoma/polyp (SSA/P). The terms "sessile serrated adenoma (SSA)," "sessile serrated polyp," and "sessile serrated lesion" are considered synonymous and are widely accepted. However, "sessile serrated adenoma" without dysplasia does not exhibit true cytological dysplasia. I am curious about "UD, undetermined" SSA used in this article. I think that UD SSA

Received June 12, 2018. Revised June 15, 2018.

Accepted June 15, 2018.

Correspondence to Hyoun Woo Kang, Department of Internal Medicine,

Dongguk University Ilsan Hospital, Dongguk University College of Medicine,

27 Dongguk-ro, Ilsandong-gu, Goyang 10326, Korea. Tel: +82-31-961-7128,

Fax: +82-31-961-9309, E-mail: gangmali@naver.com may mean SSA/P without dysplasia.

This study highlights the interest in serrated polyps with regard to recently identified malignant potential and the need for surveillance. The data used to guide surveillance strategy followed by resection of SSA/P have been limited until now. There are no high-quality prospective data currently available or are likely to be available in the next few years. This study suggested that patients with SSAs do not need to be monitored by colonoscopy annually. In determining a surveillance interval, it is important to know how long after polypectomy the advanced polyps are found. The follow-up period in their study was less than 3 years on average and thus it seems difficult to determine the proper interval for surveillance colonoscopy to prevent new CRCs. Recent guidelines recommend that the surveillance interval should be based on polyp size and histology. ${ }^{2-5}$ A U.S. MultiSociety Task Force guideline suggested 5-, 3-, and 1-year colonoscopic follow-up for SSA/P with no dysplasia, SSA/P with dysplasia or size $\geq 10 \mathrm{~mm}$, and serrated polyposis syndrome, respectively. However, prospective data to support the surveillance intervals are lacking and these recommendations are based on expert opinion.

The data in this study showed a relatively high detection rate for SSA during surveillance colonoscopy despite short interval follow-up colonoscopy (total polyps: $44.8 \%$ at 1 st follow-up, $47.4 \%$ at 2nd follow-up; SSA: $17.8 \%$ at 1st followup, $22 \%$ at 2nd follow-up). This finding may be due to missed lesions on screening colonoscopy for several reasons, as described by the authors (high-quality surveillance and resultant increase in detection rate, expertise of colonoscopists, and the extent of bowel preparation). However, incomplete resection could be another cause. The most common size of an SSA was 5 to $10 \mathrm{~mm}$ in this study, but nearly half of the

๑ Copyright 2018. Korean Association for the Study of Intestinal Diseases. All rights reserved.

This is an Open Access article distributed under the terms of the Creative Commons Attribution Non-Commercial License (http://creativecommons.org/licenses/by-nc/4.0)

which permits unrestricted non-commercial use, distribution, and reproduction in any medium, provided the original work is properly cited. 
lesions were resected using cold biopsy. Recent guidelines recommend that en bloc resection of a 6- to 9-mm colon polyp should be performed using snare polypectomy. ${ }^{6}$

In a recent Korean study, no SSA/P was detected during follow-up colonoscopy in patients who underwent endoscopic mucosal resection (mean follow-up duration, 11.8 month). ${ }^{7}$

Of note, SSAs are endoscopically characterized by a flat shape, an indiscrete edge, and color similar to the surrounding mucosa, and are often covered with mucus. These morphological features make it difficult to detect SSAs and is why we focus on the need for active surveillance in patients with SSAs. Although current screening and surveillance colonoscopy is proven to reduce CRC incidence and mortality, interval cancers following negative colonoscopy still contribute to CRC burden. Interval CRC is more likely found in the proximal colon and is related to microsatellite instability and CpG island methylator phenotype. ${ }^{3}$ These clinical and biological features of interval cancers are also characteristic of the serrated pathway of CRC, starting with serrated adenomas. ${ }^{8}$ Accordingly, a careful examination as well as an appropriate screening interval are warranted to find SSAs that can be missed and are likely to become interval cancers later. I am curious about the molecular characteristics of the 8 adenocarcinomas in this study.

In summary, Park et al. ${ }^{1}$ supported current recommendations for the surveillance interval in patients with SSAs based on the descriptive analysis of surveillance outcomes. A highquality prospective study is warranted to determine the optimal post-polypectomy surveillance protocol for SSA/P.

\section{FINANCIAL SUPPORT}

The authors received no financial support for the research, authorship, and/or publication of this article.

\section{CONFLICT OF INTEREST}

No potential conflict of interest relevant to this article was reported.

\section{AUTHOR CONTRIBUTION}

Writing, Ji Hyung Nam; review and editing, Hyoun Woo Kang; approval of final manuscript, all authors.

\section{REFERENCES}

1. Park SJ, Yoon H, Jung IS, et al. Clinical outcomes of surveillance colonoscopy for patients with sessile serrated adenoma. Intest Res 2018;16:134-141.

2. Rex DK, Ahnen DJ, Baron JA, et al. Serrated lesions of the colorectum: review and recommendations from an expert panel. Am J Gastroenterol 2012;107:1315-1329.

3. Lieberman DA, Rex DK, Winawer SJ, Giardiello FM, Johnson DA, Levin TR. Guidelines for colonoscopy surveillance after screening and polypectomy: a consensus update by the US Multi-Society Task Force on Colorectal Cancer. Gastroenterology 2012;143:844-857.

4. East JE, Atkin WS, Bateman AC, et al. British Society of Gastroenterology position statement on serrated polyps in the colon and rectum. Gut 2017;66:1181-1196.

5. Hassan C, Quintero E, Dumonceau JM, et al. Post-polypectomy colonoscopy surveillance: European Society of Gastrointestinal Endoscopy (ESGE) guideline. Endoscopy 2013;45:842-851.

6. Ferlitsch M, Moss A, Hassan C, et al. Colorectal polypectomy and endoscopic mucosal resection (EMR): European Society of Gastrointestinal Endoscopy (ESGE) clinical guideline. Endoscopy 2017;49:270-297.

7. Seo JY, Choi SH, Chun J, et al. Characteristics and outcomes of endoscopically resected colorectal cancers that arose from sessile serrated adenomas and traditional serrated adenomas. Intest Res 2016;14:270-279.

8. O’Brien MJ, Zhao Q, Yang S. Colorectal serrated pathway cancers and precursors. Histopathology 2015;66:49-65. 